Tohoku J. exp. Med., 1973, 111, 87-91

\title{
Prenatal Diagnosis of the Hurler Syndrome: Mucopolysaccharide Pattern in Amniotic Fluid
}

\author{
Kryoshi Omura, Shinobu Higami, Gen Issiki, KoJI \\ Nishtzawa and Keiya Tada \\ Department of Pediatrics, Osaka City University Medical \\ School, Osaka
}

\begin{abstract}
Omdra, K., Higami, S., Issiki, G., Nishizawa, K. and Tada, K. Prenatal Diagnosis of the Hurler Syndrome: Mucopolysaccharide Pattern in Amniotic Fluid. Tohoku J. exp. Med., 1973, 111 (1), 87-91 — Prenatal diagnosis was successfully accomplished by investigating mucopolysaccharide pattern in amniotic fluid for two women who had previously borne children affected with the Hurler syndrome. Amniotic fluid obtained at the 20th week of gestation from Case A of high risk pregnancy revealed an abnormal pattern of mucopolysaccharides, a striking increase in dermatan sulfate and heparan sulfate. Her pregnancy was terminated and the aborted fetus was proved to be affected with the Hurler syndrome. In Case B of high risk pregnancy, amniotic fluid obtained at the 20th week of gestation showed normal pattern of mucopolysaccharide. Her pregnancy was continued and resulted in the delivery of a healthy girl at full term. This chemical method for antenatal detection of the Hurler syndrome is thought to be useful and reliable in the later stage of pregnancy.—- prenatal diagnosis; Hurler syndrome
\end{abstract}

Recent emphasis in medical genetics has been focused on the prenatal detection of chromosomal aberrations and inherited metabolic deseases. The Hurler syndrome is an incurable disorder transmitted as an autosomal recessive trait, characterized biochemically by excessive intracellular accumulation and urinary excretion of dermatan sulfate and heparan sulfate, and clinically by grotesque facial appearance, mental retardation, skeletal deformities and corneal clouding.

This paper describes successful cases of prenatal diagnosis which was accomplished in two women who had previously borne children with the Hurler syndrome.

\section{Methods and Materials}

Case A, a 28-year-old woman visited to our Genetic Counseling Clinic in the 19th week of her second pregnancy. She and her husband were not consanguineous. Her first son who died at 9 months of age, was diagnosed as the Hurler syndrome by urinary excretion of mucopolysaccharide (dermatan sulfate and heparan sulfate) and autopsy findings. Transabdominal amniocentesis was performed at the 20th week of gestation.

Case B, a 29-year-old women came to our Genetic Counseling Clinic in the 18th week of her third pregnancy. She and her husband were consanguineous and produced two living children: the first child, a girl, is now 4 years old and healthy; the second child, a boy is 2 years old and exhibits the clinical and laboratory signs of the Hurler syndrome, consisting of typical grotesque facial appearance, mental retardation, skeletal deformities,

Received for publication, March, 27, 1973. 
corneal clouding and urinary excretion of dermatan sulfate and heparan sulfate. The cultured skin fibroblast from this boy showed a marked increase of ${ }^{35} \mathrm{~S}$-mucopolysaccharide which was typical pattern of mucopolysaccharidosis. Amniocentesis was performed at the 20th week of gestation for prenatal diagnosis.

Identification and quantitation of mucopolysaccharides in amniotic fluid were made principally based on Matalon and Dorfman's procedure (1972) as follows.

5-10 $\mathrm{ml}$ of amniotic fluid were adjusted at $\mathrm{pH}$ 8-9 with $0.1 \mathrm{M} \mathrm{NaOH}$ and added with $\mathrm{NaCl}$ to a concentration of $0.1 \mathrm{M}$, then was treated with $10 \mathrm{mg}$ of protease (Kaken Kagaku Co.) at $37^{\circ} \mathrm{C}$ for overnight. After $50 \%$ cold trichloroacetic acid was added one fifth and immediately centrifuged, the supernatant was dialyzed against distilled water for $24 \mathrm{hr}$. The solution was concentrated and then dissolved in $0.2 \mathrm{ml}$ of distilled water and was added with $\mathrm{NaCl}$ to a concentration of $0.04 \mathrm{M}, \mathrm{NaOH}$ to a concentration of $0.1 \mathrm{M}$ and one drop of $10 \%$ cetyltrimethylammonium bromide. The solution was allowed to stand at room temperature for 12 hours to precipitate mucopolysaccharide. The solution was centrifuged at 4,000 r.p.m. for ten minutes. To the precipitate was added 1 or 2 drops of $65 \%$ propanol and $0.8 \mathrm{ml}$ of ethanol saturated with sodium acetate, then the solution was centrifuged. The precipitate was dissolved in $0.1 \mathrm{ml}$ of distilled water. One or two $\mu \mathrm{l}$ was used for identification of polysaccharides by electrophoresis for twenty minutes in $0.05 \mathrm{M}$ veronal buffer (PH 8.8) and for two hours in $0.075 \mathrm{M}$ balium acetate and $0.075 \mathrm{M}$ calcium acetate on Separax strips (Joko Co.) at $120 \mathrm{v}$. The strips were stained with $0.5 \%$ Arcian Blue in $5 \%$ acetic acid. $20 \mu \mathrm{l}$ from the remaining solution was analyzed for uronic acid by the method of Bitter and Muir (1962). The remaining solution was divided into three parts, the mucopolysaccharides were degraded in a manner of enzymatic digestion with streptomyces hyaluronidase (Amano Co.), chondroitinase AC-II and chondroitinase ABC (Seikagaku Kogyo Co.), respectively (Ohya and Kaneko 1970), and the quantitation of each fraction of the mucopolysaccharide in amniotic fluid was measured by densitometry.

As controls, amniotic fluid obtained during the 18th to 22 nd weeks of gestation for chromosome analysis and during the 37 th to 40 th weeks of gestation for obstetrical indication was analyzed for mucopolysaccharide.

The rate of incorporation of radioactive sulfate into intracellular mucopolysaccharide by cultured skin fibroblasts were examined according to Fratantoni et al.'s procedure (1968).

\section{Results}

Table 1 summarizes the results of mucopolysaccharide analysis in seventeen samples of normal amniotic fluids obtained during the 18th to 22nd week pregnancies and 37 th to 40 th week pregnancies. The average content of mucopolysaccha-

TABLE 1. Mucopolysaccharides in normal amniotic fluid

\begin{tabular}{ccc} 
& $\begin{array}{c}\text { Amniotic fluid from } \\
\text { 18th to 22nd week gestation }\end{array}$ & $\begin{array}{c}\text { Amniotic fluid from } \\
\text { 37th to 40th week gestation }\end{array}$ \\
\hline $\begin{array}{l}\text { Number of samples } \\
\text { Total content of mucopoly- } \\
\text { saccharide } \\
\text { Range } \\
\text { Average }\end{array}$ & 7 & 10 \\
\hline $\begin{array}{c}\text { Mucopolysaccharide pattern } \\
\text { Hyaluronic acid }\end{array}$ & $0.004-0.008 \mathrm{mg} / \mathrm{ml}$ & $0.0015-0.006 \mathrm{mg} / \mathrm{ml}$ \\
Chondroitin sulfate & $0.006 \mathrm{mg} / \mathrm{ml}$ & $0.004 \mathrm{mg} / \mathrm{ml}$ \\
A and C & $86 \%$ & $76 \%$ \\
\hline
\end{tabular}

Note: The content of mucopolysaccharide was expressed as uronic acid. 
ride in the fluid was found to be approximately $0.006 \mathrm{mg}$ per ml at the 18 th to $22 \mathrm{nd}$ week pregnancies and $0.004 \mathrm{mg}$ per $\mathrm{ml}$ at the $37 \mathrm{th}$ to 40 th week pregnancies. It was found that hyaluronic acid represents $86 \%$ of the total mucopolysaccharides and the remaining mucopolysaccharide was a mixture of chondroitin sulfate $\mathrm{A}$ and $\mathrm{C}$ at the 18th to $22 \mathrm{nd}$ week pregnancies, and at the 37th to 40 th week pregnancies hyaluronic acid was found to be $76 \%$ and the remaining consisted of chondroitin sulfates A and C. No other polysaccharides were detected.

Amniotic fluid obtained from Case A of high risk pregnancy revealed an abnormal pattern of mucopolysaccharides, although the total content was only sightly increased (Table 2). A striking finding was the presence of $24 \%$ dermatan

TABLE 2. Analysis of acid mucopolysaccharide in amniotic fluid of high risk pregnancy for the Hurler syndrome

\begin{tabular}{cccccc}
\hline & $\begin{array}{c}\text { Mucopolysaccharides } \\
\mathrm{mg} / \mathrm{ml} \text { (as uronic } \\
\text { acid) }\end{array}$ & \multicolumn{4}{c}{ Distribution of polysaccharides } \\
\cline { 3 - 6 } & $\begin{array}{c}\text { Hyaluronic } \\
\text { acid }\end{array}$ & $\begin{array}{c}\text { Chondroiton } \\
\text { sulfate }\end{array}$ & $\begin{array}{c}\text { Dermatan } \\
\text { sulfate }\end{array}$ & $\begin{array}{c}\text { Heparan } \\
\text { sulfate }\end{array}$ \\
\hline $\begin{array}{c}\text { Controls (18-22W) } \\
\text { High risk pregnancy }\end{array}$ & $0.004-0.008$ & $86 \%$ & $14 \%$ & $\%$ & $\%$ \\
Case A & 0.009 & 25 & 24 & 22 & 29 \\
Case B & 0.008 & 90 & 10 & & \\
\hline
\end{tabular}

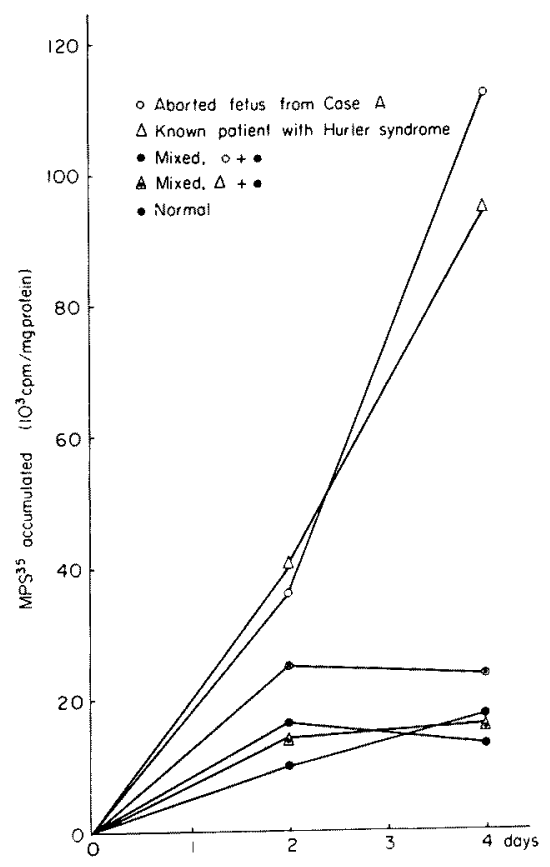

Fig. 1. Incorporation of ${ }^{35} \mathrm{SO}_{4}$ into intracellular mucopolysaccharide by cultured skin fibroblasts. 
sulfate and $29 \%$ heparan sulfate. Her pregnancy was terminated at the 24th week of gestation by her and her husband's desire. The skin fibroblasts obtained from the aborted fetus were cultured and the rate of incorporation of radioactive sulfate into intracellular mucopolysaccharide by cultured skin fibroblasts were examined. As shown in Fig. 1, fibroblasts from the aborted fetus of Case A exhibited a linear increase in MPS ${ }^{35}$ accumulation similar to that of fibroblasts from the patient with the Hurler syndrome. In case of mixed cell culture of the fibroblasts from the aborted fetus of Case A and normal fibroblasts, almost normal pattern of the MPS ${ }^{35}$ accumulation was observed, which was identical to that of mixed cell culture of the fibroblasts from the patient with the Hurler syndrome and normal fibroblasts. The liver specimen obtained from the aborted fetus showed an abnormal accumulation of dermatan sulfate and heparan sulfate on electrophoretic analysis. An accumulation of mucopolysaccharides was recognized also in the brain.

On the other hand, mucopolysaccharide pattern in amniotic fluid obtained from Case B of high risk pregnancy was found to be within normal limites. Then her pregnancy was continued and resulted in the delivery of healthy girl with a weight of $3,250 \mathrm{~g}$ at full term. At 4 weeks, the physical examinations showed a normal infant and her urine was negative for mucopolysaccharides.

\section{Comment}

Intrauterine diagnosis of the mucopolysaccharidosis has been accomplished by Fratantoni et al. (1969), by means of examining the rate of incorporation of radioactive sulfate into intracellular mucopolysaccharide in cultured amniotic fluid cells. Thereafter, Matalon and Dorfman (1972) reported a simpler and more rapid procedure with use of electrophoretic analysis of mucopolysaccharide in amniotic fluid.

According to Matalon and Dorfman (1972), an abnormal pattern of mucopolysaccharide in amniotic fluid was detected in two cases of high risk pregnancies of mucopolysaccharidosis; one was made amniocentesis at the 14th week of gestation which resulted in the delivery of an infant with the Hurler syndrome, and the other was made at the 22 nd week of gestation which resulted in abortion and was confirmed Hurler syndrome. However, two pregnancies monitored by amniotic fluid analysis, which showed normal pattern of mucopolysaccharide at the 10 th week and 16 th week of gestation, resulted in delivery of the infants affected with mucopolysaccharidosis. These results give rise to question that the analysis of mucopolysaccharide pattern in amniotic fluid was not reliable at the 16th week gestation or earlier.

Our present results suggest that this chemical method is useful and reliable at a later stage of pregnancy (at the 20th week gestation). This may be due to increase of fetus urine in amniotic fluid.

The method of examining the accumulation of ${ }^{35} \mathrm{SO}_{4}$-mucopolysaccharide in cultured amniotic fluid cells is thought to be an accurate and reliable procedure 
for antenatal diagnosis of the mucopolysaccharidosis, but it requires a fairly long time (more than 4 weeks) for culture to get sufficient amounts of cells. In the later stage of gestation when quick diagnosis is requested, this procedure is not always available. In such a case, the chemical method mentioned above will be useful.

\section{References}

1) Bitter, T. \& Muir, H.M. (1962) A modified uronic acid carbazole reaction. Analyt. Biochem., 4, 330-334.

2) Fratantoni, J.C., Hall, C.W. \& Neufeld, E.F. (1968) The defect in Hurler's and Hunter's syndromes: Faulty degradation of mucopolysaccharide. Proc. nat. Acad. Sci., 60, 699-706.

3) Fratantoni, J.C., Neufeld, E.F., Uhlendorf, W. \& Jacobson, C.B. (1969) Intrauterine diagnosis of the Hurler and Hunter syndromes. New Eng. J. Med., 280, 686688.

4) Matalon, R. \& Dorfman, A. (1972) Antenatal Diagnosis, edited by A. Dorfman, Chicago and London. The University of Chicago Press, pp. 213-216.

5) Ohya, T. \& Kaneko, Y. (1970) Novel hyaluronidase from streptomyces. Biochem. biophys. Actx, 198, 607-609. 